\title{
Avaliação da Transmissão de Televisão Digital Móvel em Redes UMTS
}

\author{
Jerônimo S. Rocha, Carlos Danilo M. Regis, Pedro Leonardo F. Costa, \\ José Ewerton P. Farias e Marcelo S. Alencar
}

\begin{abstract}
Resumo-Neste trabalho são apresentados os resultados da investigação de transmissão do serviço de televisão móvel em redes UMTS/WCDMA FDD. Para tanto, foram realizadas simulações e obtidos alguns indicadores de desempenho, como a razão entre a interferência de outras e da própria célula, fator de carga, número de usuários atendidos simultaneamente, razão portadora-interferência e potência média de transmissão das estações radiobase.
\end{abstract}

Palavras-Chave-TV móvel, UMTS, WCDMA, Desempenho de dedes UMTS

Abstract-This paper presents the results of investigation of transmission over UMTS/WCDMA FDD networks. Simulations were carried on to obtain performance estimates, including the other-to-own cell interference ratio, number of simultaneous users in the system, carrier-to-interference ratio and average transmission power of the radiobase stations.

Keywords-Mobile TV, UMTS, WCDMA, UMTS networks performance

\section{INTRODUÇÃO}

Os dispositivos móveis atuais têm acesso a diversas redes de comunicações, por exemplo redes celulares, redes $W i$-Fi (IEEE 802.11), e, mais recentemente, redes de TV digital móvel. Essas redes permitem que os usuários assistam vídeos após ou durante o download. Isso depende da taxa de transmissão da rede e do sinal de vídeo desejado.

A TV móvel é uma tecnologia que permite transmitir programas televisivos ou vídeos para dispositivos móveis, como telefones celulares e PDAs. Uma das possibilidades de exploração desse serviço é a utilização das redes de terceira geração de comunicações móveis (3G). Essas redes podem fornecer serviços com taxas de transmissão suficientes para a TV móvel.

Neste trabalho é analisada a transmissão de TV móvel em uma rede UMTS, empregando interface aérea baseada em WCDMA. Na Seção II são apresentadas algumas características de redes dessa natureza. Na Seção III é descrito o sistema de TV móvel, com maior ênfase nos aspectos necessários para transmissão em redes UMTS. Na Seção IV, são apresentados alguns indicadores de desempenho de redes UMTS, relacionados principalmente com a interferência. $\mathrm{Na}$ Seção V são apresentados os dados de entrada das simulações. Os resultados dessas simulações são mostrados e discutidos

Jerônimo Silva Rocha, Carlos Danilo Miranda Regis, Pedro Leonardo Falcão Costa, José Ewerton Pombo de Farias e Marcelo Sampaio de Alencar, Instituto de Estudos Avançados em Comunicações (Iecom), Departamento de Engenharia Elétrica, Universidade Federal de Campina Grande (UFCG), Campina Grande, Brasil, E-mails: \{jeronimo,danilo,pedro,ewerton,malencar\}@iecom.org.br. Este trabalho teve o apoio do Iecom e foi parcialmente financiado pelo $\mathrm{CNPq}$. na Seção VI. Por fim são apresentadas algumas conclusões na Seção VII.

\section{REDES UMTS BASEADAS EM WCDMA}

A terceira geração de comunicações móveis celulares é baseada em uma iniciativa da ITU (International Telecommunication Union) para estabelecer um único padrão internacional, chamado International Mobile Telecommunications - 2000 (IMT-2000). Esse conceito de padrão único evoluiu para uma família de cinco padrões 3G aprovados em maio 2001: IMTDS (Direct Sequence); IMT-MC (Multi-Carrier); IMT-TC (Time-Code); IMT-SC (Single Carrier) e IMT-FT (FrequencyTime) [1], [2]. O primeiro desses sistemas é também conhecido como UMTS (Universal Mobile Telecommunication System).

O sistema UMTS possui três partes funcionais, mostradas na Fig.1: o Equipamento do Usuário (UE); a Rede de Acesso (UTRAN - UMTS Terrestrial Radio Access Network) e a Rede Núcleo (CN) [3], [4].

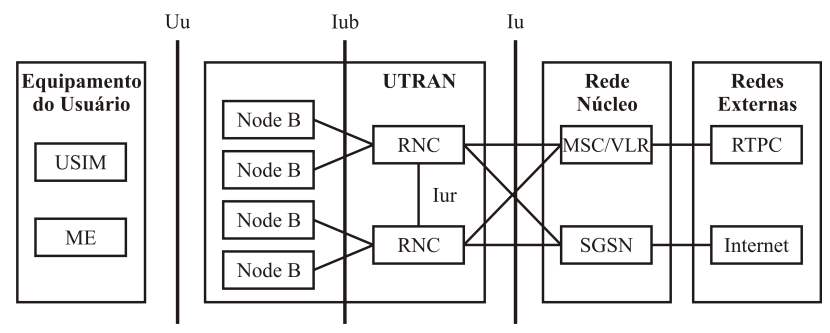

Fig. 1. Arquitetura de uma rede UMTS.

O Equipamento do Usuário é composto pelo Equipamento Móvel (ME) e pelo USIM (UMTS Subscriber Identity Module). O USIM é um cartão inteligente que contém todas as informações relevantes do usuário.

A UTRAN, por sua vez, também é formada por dois elementos distintos: a estação radiobase (ou, como é chamada nas recomendações do sistema UMTS [5], Node B) e pelo RNC (Radio Network Controller). O RNC gerencia e controla todos os recursos de rádio no seu domínio. É o RNC que realiza funções de RLC (Radio Link Control) e MAC (Medium Access Control). Várias estações radiobase estão ligadas a um único RNC que é o ponto de acesso a todos os serviços que a UTRAN provê à rede fixa. As estações radiobase, por sua vez, realizam os procedimentos da camada física [6], [1].

A Rede Núcleo é a parte que realiza o roteamento e a comutação das chamadas com conexões a redes externas como a RTPC (Rede de Telefonia Pública Comutada) ou à Internet. Ela contém o HLR (Home Location Register), o MSC (Mobile 
Services Switching Centre), o VLR (Visitor Location Register), o EIR (Equipment Identity Register), o GMSC (Gateway MSC), o SGSN (Serving GPRS Support Node) e o GGSN (Gateway GPRS Support Node).

$\mathrm{Na}$ interface entre o UE e a UTRAN é usado o Acesso Múltiplo por Divisão em Código de Faixa Larga - WCDMA (Wideband Code Division Multiple Access), de sequiência direta, definido pelo ETSI em 1998 [7]. Nesse sistema, os dados do usuário são espalhados espectralmente em toda a banda disponível, com taxa de chips de 3,84 Mchip/s. Isso resulta em uma portadora com largura de banda de aproximadamente $5 \mathrm{MHz}$.

A primeira rede 3G (baseada em WCDMA) foi lançada em Outubro de 2001 no Japão pela NTT DoCoMo, mas não funcionou comercialmente até 2003, quando outras redes $3 \mathrm{G}$ também foram lançadas em outros países [1]. O número de assinantes WCDMA, em Julho de 2007, é maior que 37 milhões no Japão e ultrapassa 115 milhões no mundo inteiro. O número de assinantes de todas as tecnologias CDMA 3G é superior a 461 milhões, incluindo CDMA2000, WCDMA e suas evoluções [8].

\section{A. Características de Sistemas WCDMA}

Há dois modos de operação WCDMA: o FDD (Frequency Division Duplex) e o TDD (Time Division Duplex) [2].

A operação das estações radiobase é assíncrona. Com isso, diferentemente do sistema IS-95, não há necessidade de uma referência de tempo global, como um GPS (Global Positioning System) [3].

Para suportar altas taxas de transmissão (até $2 \mathrm{Mbit} / \mathrm{s}$ ), o fator de espalhamento espectral usado em sistemas WCDMA é variável. A detecção no sistema WCDMA é coerente, tanto no enlace de subida quanto no de descida, baseada em símbolos piloto.

O sistema WCDMA foi desenvolvido para operar em conjunto com o GSM (Global System for Mobile Communication). Portanto, é possível efetuar handovers entre GSM e WCDMA.

Um resumo dos principais parâmetros relacionados com os sistemas WCDMA é mostrado na Tabela I.

TABELA I

PRINCIPAIS PARÂMETROS DO SISTEMA WCDMA

\begin{tabular}{|l|l|}
\hline Método de acesso múltiplo & DS-WCDMA \\
\hline Modos de Operação & FDD/TDD \\
\hline Sinc. da Estação Radiobase & Operação Assíncrona \\
\hline Taxa de chips & 3,84 Mchip/s \\
\hline Duração de um Quadro & $10 \mathrm{~ms}$ \\
\hline Multiplexação dos Serviços & $\begin{array}{l}\text { Diversos serviços com diferentes } \\
\text { QoS em uma mesma conexão }\end{array}$ \\
\hline Múltiplas Taxas & Fator de espalhamento variável \\
\hline
\end{tabular}

\section{SERVIÇOS DE TV MÓVEL}

TV móvel é uma tecnologia de transmissão de programas televisivos ou vídeos para dispositivos móveis, como telefones celulares e PDAs. O serviço de TV móvel, abrange conteúdos de vídeo, áudio e informações textuais.
A TV móvel pode ser transmitida por radiodifusão, usando redes de televisão digital ou via satélites. Há vários padrões de transmissão de TV Móvel por radiodifusão. Por exemplo:

- DVB-H - padrão desenvolvido para utilização do espectro do DVB-T [9];

- MediaFlo - tecnologia de transmissão desenvolvida pela Qualcomm [10];

- DMB - tecnologia coreana que usa o espectro VHF [11];

- One Seg - padrão que usa o mesmo espectro de freqüências do ISDB-T [12].

O One Seg é o padrão de transmissão de TV móvel adotado no Brasil [13].

Os requisitos da transmissão de TV móvel são:

- transmissão em formatos adequados para dispositivos móveis, por exemplo, QCIF, CIF ou QVGA;

- baixo consumo de energia;

- recepção estável com mobilidade, para velocidades de até $250 \mathrm{~km} / \mathrm{h}$

- imagem com boa qualidade, mesmo com intenso efeito de múltiplos percursos;

A largura de banda das redes $3 \mathrm{G}$ possibilita a transmissão de vários tipos de informação multimídia, incluindo vídeo em tempo real, vídeo-conferência, áudio de alta qualidade, acesso à Internet e download de arquivos.

O sistema UMTS foi concebido para proporcionar taxas de transmissão de dados maiores que nas gerações anteriores de comunicações móveis celulares. Essas taxas aliadas às eficientes técnicas de codificação de vídeos, como MPEG-4 e H.264, possibilitam transmissão de vídeos de boa qualidade nas redes WCDMA.

\section{A. Taxa de Transmissão em Redes WCDMA}

Para transmissão de TV móvel é necessário que os vídeos sejam adaptados às características dos receptores portáteis. Essas modificações, além de melhorar a visualização do vídeo nos receptores, diminuem a taxa de transmissão, que é um fator limitante desse serviço. Um padrão de codificação de vídeo com um grande poder de compressão é o H.264, que é o sistema de codificação escolhido para o padrão brasileiro de televisão digital [14].

As taxas de transmissão utilizadas dependem da resolução espacial e temporal do vídeo e do perfil de codificação. O padrão brasileiro de TV digital define os níveis de codificação H.264 como: 1, 1b, 1.1, 1.2 e 1.3 [15]. Com o nível 1.b, pode-se utilizar vídeos QCIF com taxa de $128 \mathrm{kbit} / \mathrm{s}$ e taxa de quadros de 15 quadros/s e com o nível 1.1, vídeos QCIF com taxa de $192 \mathrm{kbit} / \mathrm{s}$ e taxa de quadros de 30 quadros/s [11].

Os sinais de TV móvel são formados pela composição de sinais de vídeo, áudio (tipicamente com $52 \mathrm{kbit} / \mathrm{s}$ ), dados de sincronização e sinalização ( $29.25 \mathrm{kbits} / \mathrm{s})$ e dados com informações do conteúdo dos programas transmitidos (40 kbits/s). A taxa resultante dos sinais desse serviço é a soma das taxas dos sinais que formam o sinal resultante. A taxa mais usada em redes WCDMA para transmissão de vídeos é $384 \mathrm{kbit} / \mathrm{s}$, para vídeo QVGA (320x240) [11], [16]. 


\section{ANÁlise dA INTERFERÊNCIA}

Em redes WCDMA é usada a mesma portadora em várias células da rede, de forma que os usuários são separados apenas pelas sequências de códigos usadas no espalhamento espectral. O desempenho de um sistema WCDMA é então comprometido fortemente pela interferência, que limita o número máximo de usuários servidos simultaneamente (capacidade da rede) e, portanto, também limita a vazão na rede [4].

Neste trabalho, a capacidade do sistema é definida como o número máximo de usuários suportados simultaneamente pelo sistema, com uma probabilidade de serviço alvo pré-definida ou, correspondentemente, com a carga alvo nos enlaces de subida e descida.

Para medir a qualidade das conexões é utilizada a relação portadora-interferência $\left(\gamma_{j k}\right)$, medida na estação móvel $j$ conectada à estação radiobase $k$. No enlace de descida, a razão portadora-interferência média nas células $(\bar{\gamma})$ é dada por

$$
\bar{\gamma}=\frac{1}{C} \frac{1}{M_{k}} \sum_{k \in C} \sum_{j \in M_{k}} \frac{L_{j k} P_{j k}}{L_{j k} \bar{\alpha}_{j}\left(\bar{P}_{k}-\nu_{j} P_{j k}\right)+\sum_{i \neq k} L_{i j} \bar{P}_{i}+N_{j}}, \text { (1) }
$$

em que, $P_{j k}$ é a potência do sinal transmitido pela estação radiobase $k$ para a estação móvel $j, L_{j k}$ é a perda de propagação sofrida pelo sinal recebido pela estação móvel, $\bar{\alpha}_{j}$ é o fator de ortogonalidade dos sinais, $\bar{P}_{k}$ é a potência média de transmissão da estação radiobase $k, \nu_{j}$ é o fator de atividade e $N_{j}$ é a potência do ruído recebido pela estação móvel $j$ [2]. $C$ é o conjunto das células na rede e $M_{k}$ é o conjunto das estações móveis na célula $k$.

A potência de transmissão das estações móveis é baseada na sensibilidade da estação radiobase, na taxa de dados, velocidade da estação móvel e perdas de percurso entre a estação móvel e a estação radiobase. $\mathrm{O}$ ajuste da potência de transmissão também leva em consideração o fator de atividade de serviço, o ganho de soft handover e o aumento médio de potência decorrente do controle rápido de potência. A sensibilidade da estação radiobase $(C T)$, também chamada de limiar de cobertura (Coverage Threshold), é dada por

$$
C T=\frac{N}{\left(1+\frac{W}{\nu_{U L} \cdot E_{b} / N_{0} \cdot R}\right)(1-\eta)},
$$

em que, $N$ é a potência do ruído na célula vazia, $W$ é a taxa de chip, $R$ é a taxa média de dados, $E_{b} / N_{0}$ a razão sinal ruído da célula, $\nu_{U L}$ é o fator de atividade de serviço no enlace de subida e $\eta$ é o fator de carga.

O fator de carga $\eta$ na célula depende da quantidade de usuários $(M)$ conectados à célula e da relação $(I)$ entre a interferência causada por usuários de outras células $\left(I_{o}\right)$ e a interferência causada pelos usuários da célula sob observação $\left(I_{p}\right)$. Dessa forma, $\eta$ pode ser expresso por

$$
\begin{aligned}
& \eta=\frac{(M-1)}{\frac{W}{R \cdot E_{b} / N_{0}(1+I)}} \\
& \text { V. SiMULAÇÕES }
\end{aligned}
$$

Para as análises da interferência em redes WCDMA na transmissão da TV móvel, foram realizadas simulações estáticas de uma rede na cidade de Espoo, na Finlândia [17]. Esse cenário foi escolhido pela sua disponibilidade e por permitir comparação com outros trabalhos. Os resultados são a média de 50 (cinqüenta) simulações estáticas.

Nas simulações, os equipamentos dos usuários foram alocados pseudo-aleatoriamente na área da rede, com distribuição uniforme, com várias densidades de usuários.

Foram escolhidas três possibilidades de qualidade de serviço para transmissão de sinais de TV móvel. Para comparação de resultados, também foram realizadas simulações de transmissão de voz. As taxas de cada um dos serviços foram atribuídas conforme a Tabela II. Deve ser observado que para TV móvel, as transmissões são assimétricas, dado que durante uma transmissão de TV móvel, é pequena a quantidade de informações no enlace de subida. As taxas nesse enlace foram mantidas em $12,2 \mathrm{kbit} / \mathrm{s}$.

TABELA II

TAXAS DE DADOS USADAS NAS SIMULAÇÕES.

\begin{tabular}{|l|c|c|}
\hline & \multicolumn{2}{|c|}{ Taxa no Enlace (kbit/s) } \\
\hline Serviço & Subida & Descida \\
\hline Voz & 12,2 & 12,2 \\
\hline TV móvel Q1 & 12,2 & 128,0 \\
\hline TV móvel Q2 & 12,2 & 256,0 \\
\hline TV móvel Q3 & 12,2 & 384,0 \\
\hline
\end{tabular}

Alguns dados de entrada do simulador são apresentados na Tabela III.

TABELA III

PARAMETROS DE SIMULAÇÃO

\begin{tabular}{|l|l|}
\hline Estação Base & $43 \mathrm{dBm}$ \\
\hline Pot. max. transmissor & $30 \mathrm{dBm}$ \\
\hline Pot. max. transmissor por Link & $30 \mathrm{dBm}$ \\
\hline Pot. do canal piloto & $20-25 \mathrm{~m}$ \\
\hline Altura da antena & $65^{\circ} / 17 \mathrm{dBi}$ \\
\hline Antenas & $75 \%$ \\
\hline Carga do uplink & $21 \mathrm{dBm}$ \\
\hline Estação Móvel & $65 \mathrm{~dB}$ \\
\hline Pot. max. transmissor & Omni / $1.5 \mathrm{dBi}$ \\
\hline Faixa dinâmica & \multicolumn{2}{|l|}{} \\
\hline Antenas & ITU Pedestrian A \\
\hline Outros & $3 \mathrm{~km} / \mathrm{h}$ \\
\hline Perfil do canal & Traçado de Raios \\
\hline Velocidade das Est. Móveis & $5 \mathrm{~dB}$ \\
\hline Modelo a perda de percurso & $144 \mathrm{~km}^{2}$ \\
\hline Margem de handover & 57 \\
\hline Área da Rede &
\end{tabular}

\section{RESUltados}

Os resultados mostrados nesta seção são apresentados em função da densidade de usuários na rede $\left(n_{U}\right)$, e das taxas de transmissão dos serviços relacionados neste trabalho.

A razão $(I)$ entre a interferência causada por usuários de outras células e a interferência causada pelos usuários da célula sob observação é mostrada na Figura 2. Nessa figura é possível notar que à medida que a densidade de usuários aumenta, a razão $I$ diminui até estabilizar. Essa diminuição pode ser atribuída ao aumento da interferência dentro da célula sob observação, que é mais intenso do que o aumento da 
interferência recebida de outras células. Percebe-se também que os valores em que $I$ se estabiliza, são maiores para menores taxas de transmissão. Dessa forma, $I$ diminui com o aumento da qualidade de transmissão do serviço de TV móvel, sendo menor para a melhor qualidade desse serviço (Q3) e maior para o serviço de voz.

A discrepância entre os valores de $I$ para os serviços de voz e TV móvel se deve à diferença na quantidade de usuários ativos na rede para esses serviços. No caso do serviço de voz, o número de usuários ativos nas células é significativamente maior que o número de usuários ativos para TV móvel, como mostrado na Figura 3. Essa diferença faz com que interferência gerada por usuários da célula sob observação, cresça mais rapidamente que a interferência proveniente de outras células, para o serviço de TV móvel.

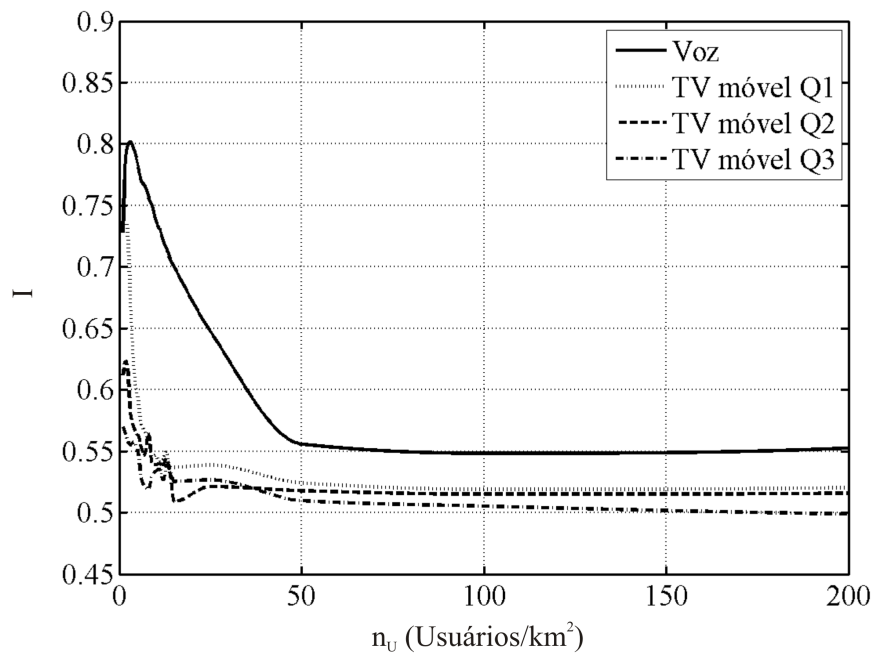

Fig. 2. Razão entre a interferência de outras células e a da própria célula.

A potência média de transmissão $\left(P_{T}\right)$ por canal de tráfego, das estações radiobase é mostrada na Figura 4. Nota-se que para crescentes densidades de usuários a potência de transmissão da estação radiobase aumenta até um nível de saturação. A potência de transmissão aumenta com o número de usuários ativos na célula [2], [4]. Nas simulações, o número médio de usuários ativos nas células satura em determinado nível, como mostrado na Figura 3. Isso explica a saturação nas curvas da potência média de transmissão das estações radiobase.

Pode-se observar que o número máximo de usuários atendidos simultaneamente cresce para pequenas densidades de usuários na área de cobertura da rede, mas estabiliza logo em seguida para os serviços de TV móvel. Isso limita a vazão da rede no enlace de descida. Esse efeito é mais crítico para crescentes taxas de transmissão da TV móvel.

A relação portadora-interferência média $\bar{\gamma}$ nas células aumenta com a taxa de transmissão e aumenta também com o número de usuários atendidos simultaneamente nas células. Como o número de usuários atendidos satura, a relação $\bar{\gamma}$ também satura, como pode ser visto na Figura 5.

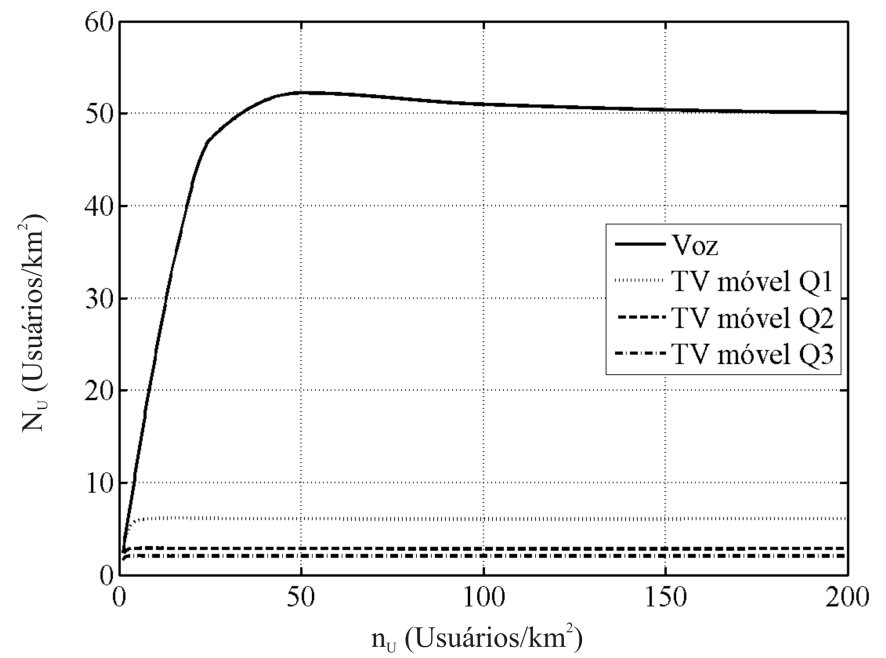

Fig. 3. Número médio de usuários servidos por célula.

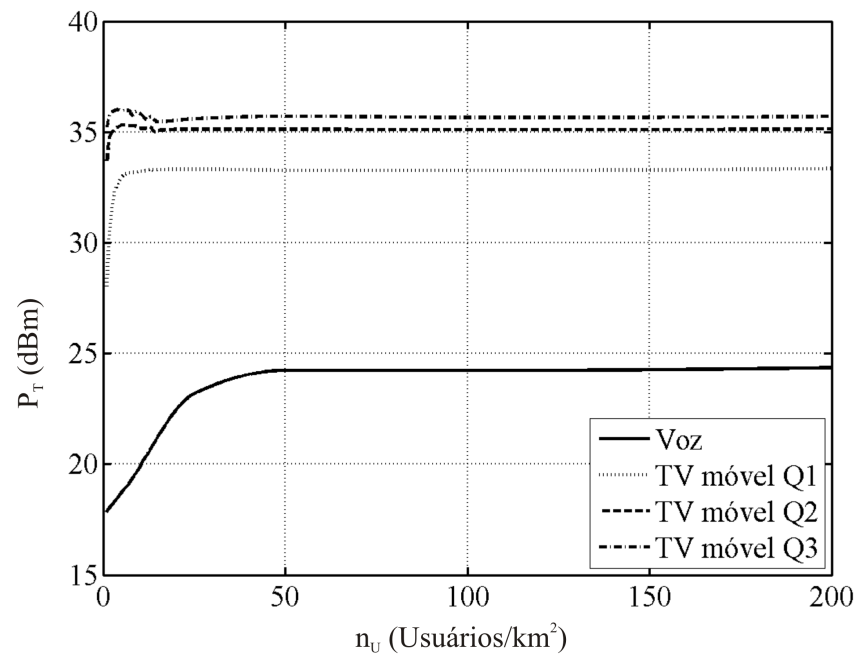

Fig. 4. Potência de transmissão das estações radiobase.

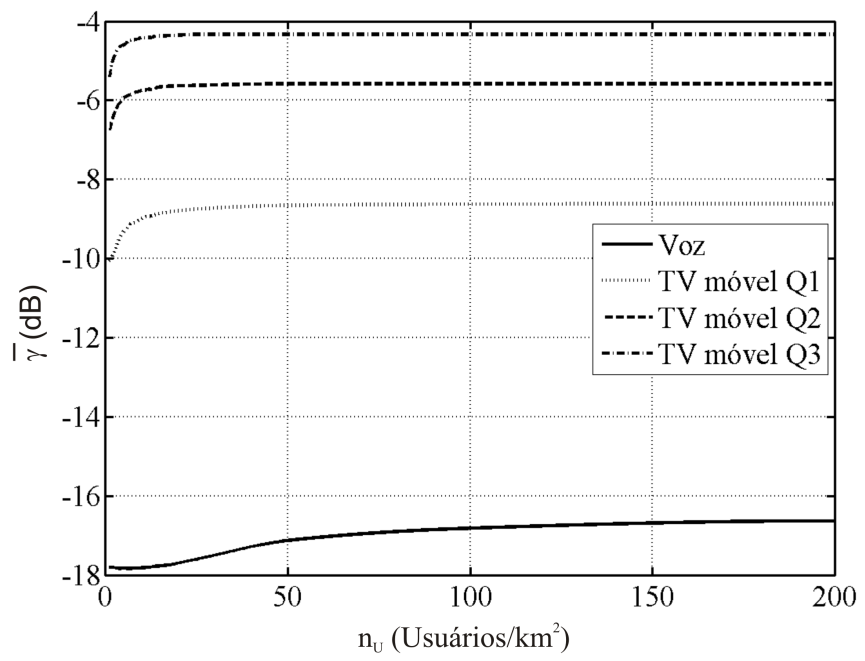

Fig. 5. Relação portadora-interferência média nas células. 


\section{CONCLUSÕES}

Neste trabalho é analisado o desempenho do sistema UMTS/WCDMA para transmissão de TV móvel com três qualidades de serviço diferentes. Para essa análise foram usadas simulações estáticas de uma rede com cenário em Espoo, na Finlândia.

Analisou-se a razão média entre a interferência proveniente de outras células e a interferência da própria célula. Essa razão se mostrou decrescente com a densidade de usuários, até um nível mínimo em que se estabiliza. Esse nível depende do tipo de serviço oferecido e é menor para mais altas qualidades de transmissão da TV móvel.

Analisou-se também a variação da razão portadorainterferência média nas células, com relação à densidade de usuários e à taxa de transmissão da TV móvel. Os resultados indicam que, para maiores taxas de transmissão, essa razão deve também ser maior. Além disso, verificou-se pequena variação em relação à densidade de usuários para cada taxa de transmissão de dados.

Para altas qualidades do serviço de TV móvel, ou seja, maiores taxas de transmissão, a quantidade de usuários atendidos diminui. Para o serviço de TV móvel com qualidade Q1 (128 kbit/s), a quantidade máxima de usuários ficou em torno de seis usuários por célula. Para os serviços com qualidade Q2 e Q3 (256 kbit/s e $384 \mathrm{kbit} / \mathrm{s})$, respectivamente, o número de usuários servidos simultaneamente nas células foi aproximadamente dois e três. Esses números estão bastante abaixo do número médio de usuários de voz.

\section{REFERÊNCIAS}

[1] P. Lescuyer, UMTS: Origins, Architecture and the Standard. Springer, 2002.

[2] M. J. Nawrocki, M. Dohler, and A. H. Aghvami, Understanding UMTS Radio Network Modelling, Planning and Automated Optimisation. John Wiley \& Sons, 2006.

[3] H. Holma and A. Toskala, WCDMA for UMTS: Radio Access for Third Generation Mobile Communications, 3rd ed. John Wiley \& Sons, 2004

[4] J. Laiho, A. Wacher, and T. Novosad, Radio Network Planning and Optimisation for UMTS, 2nd ed. John Wiley \& Sons, 2005.

[5] ETSI, "Digital cellular telecommunications system (phase 2+); general packet radio service (GPRS); overall description of the GPRS radio interface," European Telecommunications Standards Institute," Relatório Técnico ETSI TS 101 350, Junho 2000.

[6] B. Walke, P. Seidenberg, and M. P. Althoff, UMTS: The Fundamentals. Wiley, 2003.

[7] H. Kaaranen, A. Ahtiainen, L. Laitinen, S. Naghian, and V. Niemi, UMTS Networks: Architecture, Mobility and Services. John Wiley \& Sons, 2005.

[8] GSA, "Presentation from GSM $\rightarrow$ 3G CEE 2007," http://www.gsacom.com, visitado em 09 de Outubro de 2007.

[9] U. Reimers, DVB - The Family of International Standards for Digital Video Broadcasting. Springer, 2005.

[10] V. Friström and T. Haantie., "MOBILE TV," Innovation in Telecommunications, Proceedings of the Research Seminar on Telecommunications Business, 2006.

[11] A. Kumar, Mobile TV: DVB-H, DMB, $3 G$ Systems and Rich Media Applications. Focal Press Media tecnology Professional, 2007.

[12] ARIB, “ARIB Standard B31,” November 2005.

[13] ABNT, "NBR 15604-1 Televisão Digital Terrestre - Receptores," 2007.

[14] M. S. de Alencar, Televisão Digital. São Paulo: Editora Érica, 2007.

[15] ABNT, "NBR 15602-1 Televisão Digital Terrestre - Codificação de Vídeo, Áudio e Multiplexação," 2007.

[16] K. Matsumura, K. Sakaki, K. Otsuki, H. Kokubun, and K. Fujisawa., "A practical experiment on mobile data broadcasting," Consumer Communications and Networking Conference, 2005. CCNC. 2005 Second IEEE, pp. 337-342, Janeiro 2005.

[17] J. Laiho, A. Wacker, and T. Novosad, "Radio network planning and optimisation for UMTS - supplementary material," http://www.wiley.com/go/laiho/, visitado em 10 de maio de 2007. 